\title{
Sirtuin 3: A Janus face in cancer (Review)
}

\author{
YANLU XIONG $^{1}$, MINGXING WANG $^{1}$, JINBO ZHAO $^{1}$, YONG HAN $^{1}$ and LINTAO JIA ${ }^{2}$ \\ ${ }^{1}$ Department of Thoracic Surgery, Tangdu Hospital, Fourth Military Medical University, Xi'an, Shaanxi 710038; \\ ${ }^{2}$ State Key Laboratory of Cancer Biology, Department of Biochemistry and Molecular Biology, \\ Fourth Military Medical University, Xi'an, Shaanxi 710032, P.R. China
}

Received September 14, 2016; Accepted November 3, 2016

DOI: $10.3892 /$ ijo.2016.3767

\begin{abstract}
The NAD-dependent protein deacetylase sirtuin 3 (SIRT3) is an enzyme localized primarily in the mitochondrion, where it modulates cellular functions such as nutrient metabolism, ATP balance, antioxidant machinery, and other mechanisms fundamental to mitochondria. SIRT3 is closely associated with the pathogenesis of diverse disorders. In particular, it plays a dual role in the development and progression of cancer. In many cancers, SIRT3 acts as an oncogene by promoting or maintaining the malignant phenotypes of neoplastic cells, including uncontrolled proliferation, resistance to apoptosis, and increased motility or invasiveness; however, SIRT3 suppresses these phenotypes in certain types of malignancy. The underlying mechanisms involve depletion of intracellular reactive oxygen species, modulation of metabolic reprogramming, and regulation of intracellular signaling responsible for cell growth and death. This review summarizes recent findings concerning the characteristics and substrates/interacting partners of SIRT3, with particular emphasis on emerging mechanisms responsible for fine-tuning cellular behaviors that potentially underlie its conflicting roles in carcinogenesis.
\end{abstract}

\section{Contents}

1. Introduction

2. Protein structure and localization of SIRT3

3. Cellular events regulated by SIRT3

4. Dual role of SIRT3 in cancer

Correspondence to: Dr Lintao Jia, State Key Laboratory of Cancer Biology, Department of Biochemistry and Molecular Biology, Fourth Military Medical University, 169 Changle West Road, Xi'an, Shaanxi 710032, P.R. China

E-mail: jialth@fmmu.edu.cn

Dr Yong Han, Department of Thoracic Surgery, Tangdu Hospital, Fourth Military Medical University, 1 Xinsi Road, Baqiao, Xi'an, Shaanxi 710038, P.R. China

E-mail: hanyong_td@163.com

Key words: sirtuin 3, deacetylase, cancer, mechanism
5. Mechanisms underlying SIRT3-mediated regulation of cancer 6. Conclusion

\section{Introduction}

Cancer is one of the leading causes of mortality, accounting for $\sim 13 \%$ of deaths worldwide (1). Carcinogenesis is characterized by the enrichment of cells displaying phenotypes that result in excessive proliferation, resistance to apoptosis, and metabolic dysregulation (2). The cellular machineries that determine these cellular behaviors remain unclear. However, it is known that post-translational modification of proteins is a common mechanism responsible for fine-tuning intracellular signaling and metabolic pathways that either facilitate or impair malignant transformation.

Lysine acetylation is a fundamental modification that profoundly affects the activity of a protein, as evidenced by proteomic studies revealing that $>2,000$ proteins involved in diverse cellular processes are acetylated (3-5). The lysine acetylation status of individual proteins is concurrently determined by the balance between acetylation and deacetylation, a process catalyzed by a cohort of acetyltransferases and deacetylases, respectively. In contrast to the largely constitutive and nonselective activities of several known acetyltransferases, the mechanism of protein deacetylation has been studied extensively, leading to the characterization of four classes of deacetylase, all of which show variable catalytic characteristics and substrate profiles. Sirtuins (SIRTs), a family comprising seven members (SIRT 1-7), are class III deacetylases that deacetylate diverse histone or non-histone proteins in a nicotinamide adenine dinucleotide (NAD)-dependent manner. SIRTs are involved in regulating diverse cellular activities such as cell proliferation, survival, and metabolism (6-9). Among this evolutionarily conserved deacetylase family, SIRT3 is a primary mitochondrial deacetylase, whose dysregulation is involved in the development of diseases such as diabetes (10), myocardial injury (11), and cancer $(12,13)$. In particular, SIRT3 plays a conflicting role in cancer initiation and progression; the signaling networks involved in these processes remain unclear (13-16).

\section{Protein structure and localization of SIRT3}

The SIRT3 protein comprises two main functional domains. The larger Rossmann domain provides sites for $\mathrm{NAD}^{+}$ 
binding, whereas the smaller domain binds zinc atoms. The cleft between the two domains serves as a docking site for acetylation substrates $(17,18)$. SIRT3 is first expressed in the cytoplasm as a 399 -amino acid (44 kDa) inactive precursor. This precursor is subsequently targeted to the mitochondrion, where it is cleaved at the N-terminus by the mitochondrial matrix processing peptidase protein (MPP) to generate a mature $28-\mathrm{kDa}$ protein comprising 257 amino acid residues (19-21). Mitochondrial trafficking depends on the N-terminal 100 amino acids, of which residues 1-25 play an important role both in mitochondrial targeting and proteolytic processing (19). Intriguingly, full-length SIRT3 also exists in the nucleus, where it normally exhibits histone deacetylation activity and undergoes cytoplasmic translocation under stress conditions (22) (Fig. 1).

\section{Cellular events regulated by SIRT3}

SIRT3 is the primary mitochondrial deacetylase that determines the acetylation level of many mitochondrial proteins (23). As a result, SIRT3 plays a pivotal role in varied cellular events such as nutrient metabolism, ATP balance, antioxidant machinery regulation, and some fundamental mitochondrial functions (Fig. 2).

Nutrient metabolism. SIRT3 orchestrates global shifts in nutrient metabolism by regulating the activity of diverse substrates involved in multiple metabolic pathways. In carbohydrate metabolism, for example, SIRT3 deacetylates and inactivates cyclophilin D (CyP-D), resulting in the dissociation of hexokinase 2 (HK2) from the mitochondrial outer membrane and the subsequent inhibition of glycolysis (24). SIRT3 can also block the malate-aspartate shuttle by inhibiting glutamate oxaloacetate transaminase 2 (GOT2) (25). Acetyl-CoA is a primary entry point in the tricarboxylic acid cycle (TCA). To this end, SIRT3 can deacetylate and activate acetyl-CoA synthetase 2 (AceCS2), thereby providing increased acetyl-CoA to the TCA $(26,27)$. SIRT3 also increases acetyl-CoA levels by deacetylating the upstream enzymes responsible for activating the pyruvate dehydrogenase complex (PDC) (28). In addition, SIRT3 accelerates the oxidation of isocitrate by targeting isocitrate dehydrogenase 2 (IDH2), which plays a vital role in glutathione antioxidant systems by generating NADPH (29). Furthermore, SIRT3 coordinates the TCA cycle and the electron transport chain (ETC) by activating complex II, e.g., the flavoprotein subunit of succinate dehydrogenase (SDH) that plays a critical role in both of these processes $(30,31)$. Finally, SIRT3 interacts with complexes I, III, and IV, thereby improving the efficiency of electron transport $(10,23)$.

SIRT3 participates in amino acid metabolism and in the urea cycle. SIRT3 deacetylates and increases the enzymatic activity of glutamate dehydrogenase (GDH), a key enzyme involved in amino acid oxidation (32). Moreover, SIRT3 deacetylates ornithine transcarbamylase (OTC), thereby increasing urea cycle flux during energy restriction (33).

During lipid metabolism, SIRT3 accelerates the process of fatty acid oxidation by modulating the activity of long chain acyl-CoA dehydrogenase (LCAD) (34). SIRT3 also enhances the enzymatic activity of 3-hydroxy-3-methylglutaryl CoA synthase 2 (HMGCS2) to promote synthesis of the ketone body, $\beta$-hydroxybutyrate (35).

Antioxidant machinery and ATP balance. SIRT3 acts as a watchdog that maintains redox homeostasis by regulating the antioxidant machinery and ATP balance. For instance, SIRT3 deacetylates and activates the transcriptional factor forkhead box O3 (FOXO3a), leading to upregulation of manganese-dependent superoxide dismutase 2 (MnSOD2) and catalase (Cat), two important antioxidant proteins that deplete intracellular reactive oxygen species (ROS) (36). As mentioned above, SIRT3 also licenses glutathione antioxidant systems by targeting IDH2 (29), and facilitates electron transportation by preventing slow ETC flux-triggered electron leaking and ROS production (23). SIRT3 also interacts with and deacetylates liver kinase B1 (LKB1). The deacetylation of LKB1 activates 5' AMP-activated protein kinase (AMPK), leading to high levels of ATP (37). AMPK in turn increases SIRT3 activity by increasing cellular NAD ${ }^{+}$levels via feedback regulation (38). Furthermore, the activity of SIRT3 depends on a high ratio of cellular $\mathrm{NAD}^{+}$to NADH, an indication of energy-deficient state that increases AMPK activity $(38,39)$.

Functions of mitochondria. SIRT3 plays a fundamental role in mitochondrial functions such as repair, respiration, and dynamics. For example, SIRT3 deacetylates and prevents the degradation of 8-oxoguanine glycosylase 1 (OGG1), a DNA glycosylase enzyme responsible for repair of mitochondrial DNA (40). SIRT3 also regulates mitochondrial respiration by targeting mitochondrial ribosomal protein 10 (MRPL10) (41). In addition, SIRT3 deacetylates and activates optic atrophy 1 (OPA1), thereby modulating mitochondrial dynamics (42). SIRT3 also plays a role in maintaining mitochondrial permeability transition and preventing accumulation of misfolded proteins within mitochondria [the so-called mitochondrial unfolded protein response (UPRmt)] $(43,44)$.

\section{Dual role of SIRT3 in cancer}

The causal relationship between SIRT3 deregulation and cancer is well documented. However, SIRT3 may exhibit tumor-promoting or -suppressive capacity depending upon the type of cancer and, probably, the context of intracellular signal pathways (Fig. 3). SIRT3 also plays conflicting roles in malignancies originating from the same types of tissue, e.g., gastric cancer $(45,46)$, lung cancer $(47,48)$, colon cancer $(49,50)$, and head and neck squamous cell cancer (51-53).

SIRT3 as an oncogenic protein. SIRT3 is an oncogenic factor for many types of carcinoma: high expression correlates with hallmarks of malignancy and a poor clinical prognosis. This is exemplified by esophageal cancer, in which high expression of SIRT3 is associated with a poor outcome; indeed, the level of SIRT3 expression is an independent predictor for cancer prognosis (54). High SIRT3 expression is also associated with poor prognosis in patients with grade 3 breast cancer; SIRT3 knockdown increases the efficacy of cisplatin and tamoxifen, which are used to treat this disease (55). SIRT3 is also overexpressed in oral squamous cell carcinoma cells. Silencing of SIRT3 inhibits cancer cell proliferation, 
A

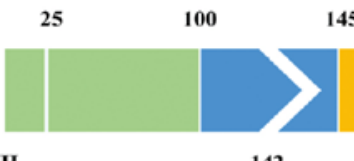

$145 \quad 228$

228

$\begin{array}{lllll}256 & 283 & 319 & 344\end{array}$

$\mathrm{NH}_{2}$

142

248

COOH

$\mathrm{NAD}^{+}$binding site

Active site

$\mathrm{Zn}^{2+}$ binding site

Mitochondrial targeting sequence

B

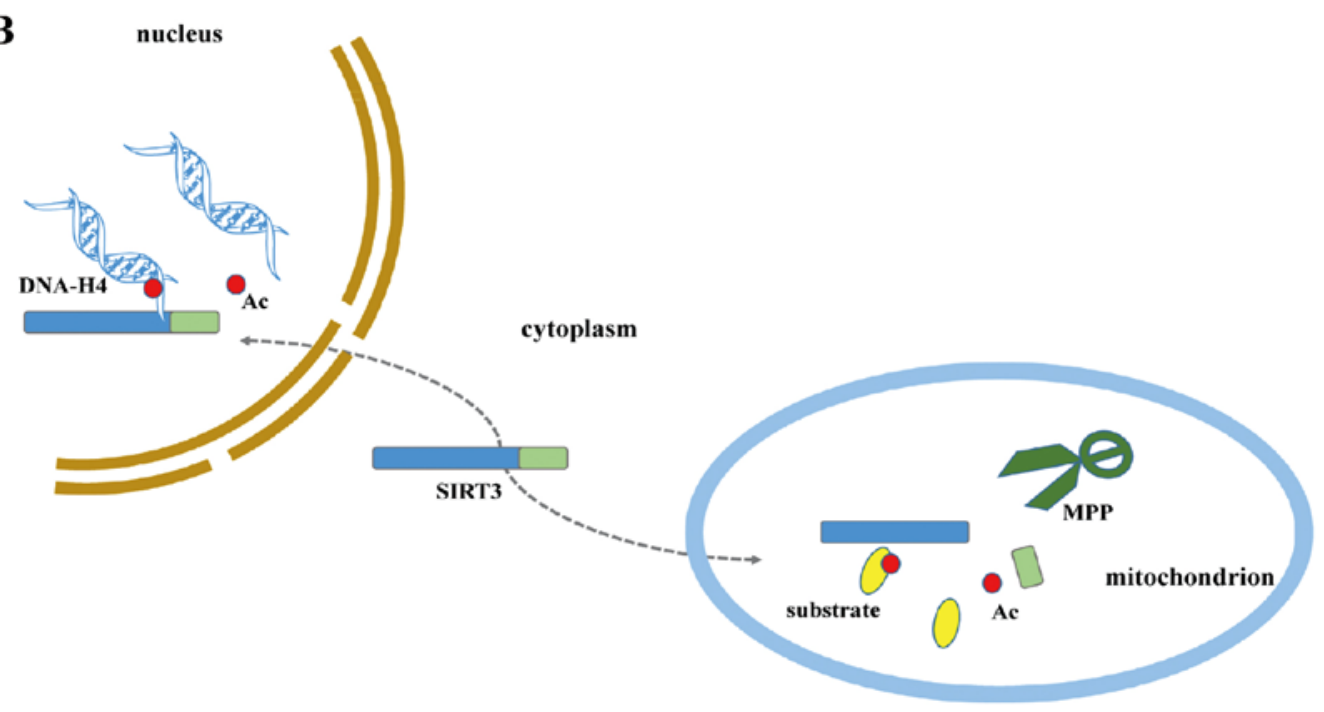

Figure 1. Structure and subcellular localization of SIRT3. (A) SIRT3 is a conserved protein belonging to the sirtuin family. SIRT3 contains an active site, four $\mathrm{NAD}^{+}$sites, four $\mathrm{Zn}^{2+}$ sites, and a mitochondrial targeting sequence. (B) SIRT3 is directed to the mitochondrion by the targeting sequence, where it undergoes cleavage by mitochondrial MPP to generate the mature protein. It functions primarily as a mitochondrial deacetylase, but also has histone deacetylation activity in the nucleus. Ac, acetyl; $\mathrm{H} 4$, histone $\mathrm{H} 4$.

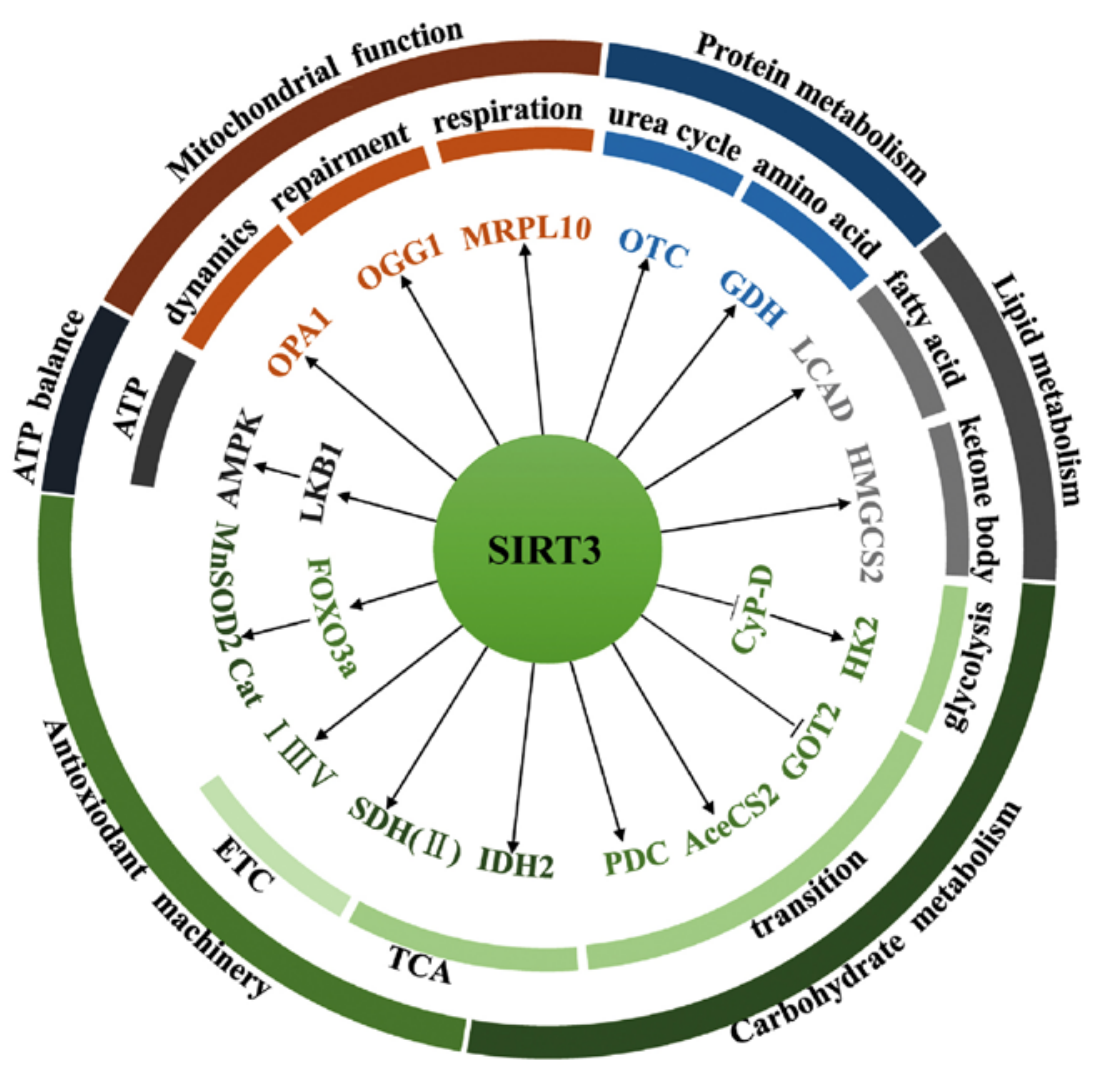

Figure 2. Functions of SIRT3 in the cell. SIRT3 is the primary mitochondrial deacetylase, mediating diverse cellular processes via deacetylation of a variety of proteins. It regulates the metabolism of carbohydrates, lipids, and amino acids by targeting critical enzymes; it functions as part of the antioxidant machinery and regulates ATP balance in different ways; it also plays a vital role in fundamental mitochondrial functions. 


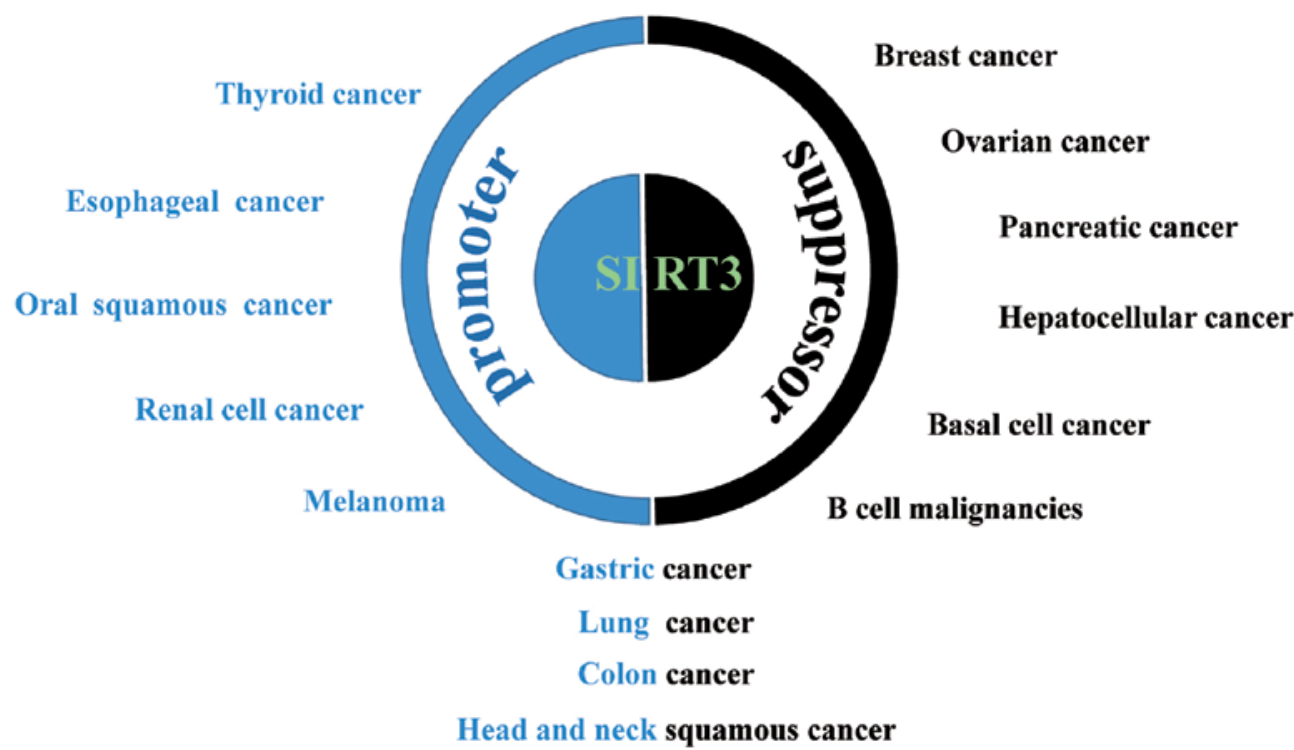

Figure 3. Dual roles of SIRT3 in cancer. SIRT3 plays conflicting roles in carcinogenesis dependent on the tissue of origin. SIRT3 functions as an oncoprotein in thyroid, esophageal, melanoma, renal, and oral squamous cancer, while acting as a tumor suppressor in breast, pancreatic, hepatocellular, ovarian, and basal cell cancers and B cell malignancies. SIRT3 can either promote or antagonize tumor development in gastric cancer, lung cancer, colon cancer, and head and neck squamous cancer.

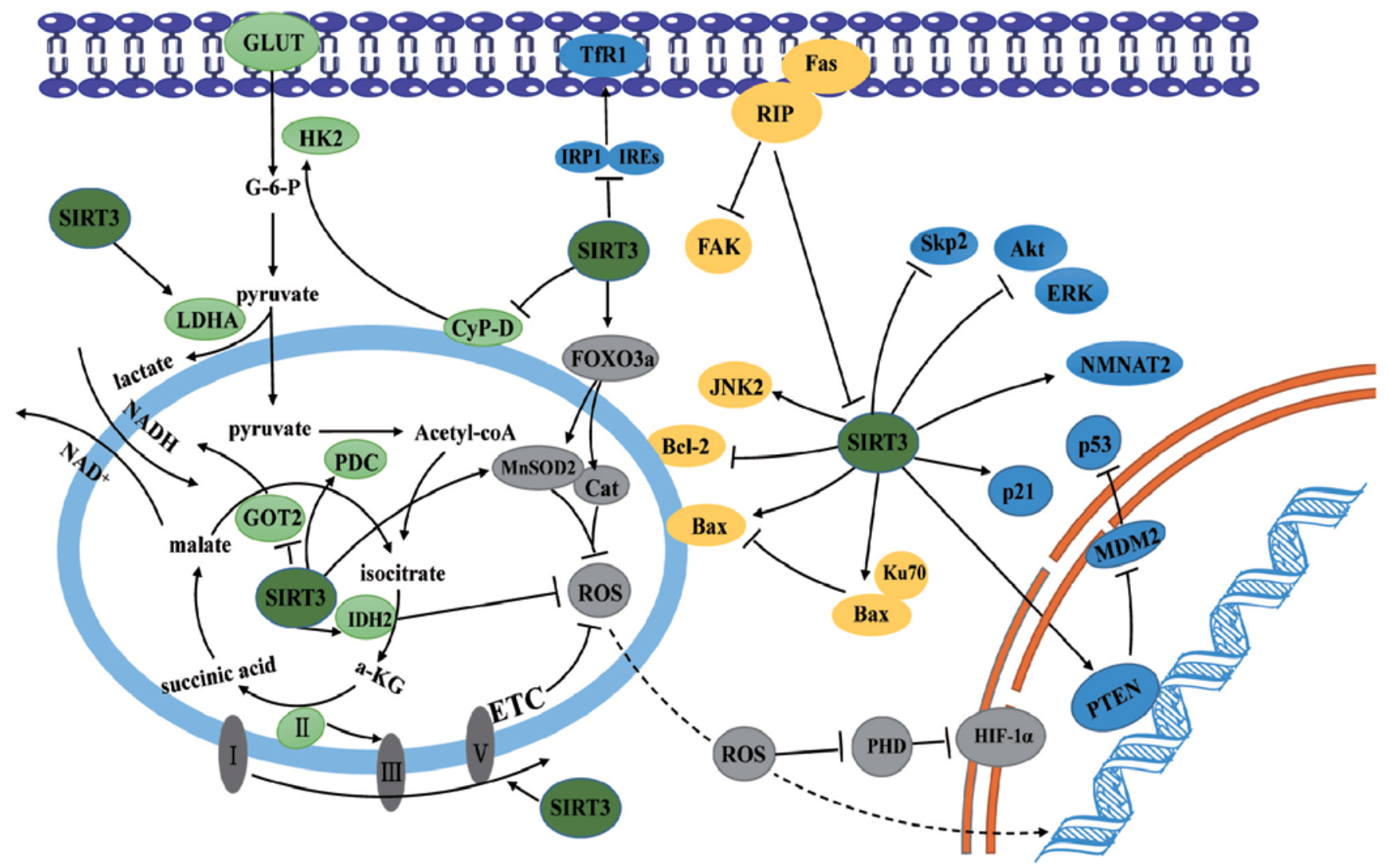

Figure 4. Mechanisms underlying the roles of SIRT3 in cancer. SIRT3 can deacetylate and regulate numerous substrate proteins involved in varied signaling events responsible for cell growth and metabolism. The regulatory role of SIRT3 in carcinogenesis relies largely on its critical involvement in the machineries determining the generation of ROS and the metabolic patterns of nutrients like carbohydrates, as well as in signaling pathways responsible for cell proliferation and apoptotic cell death.

reduces tumor burden, and sensitizes these cells to radiation or chemotherapy (56). Furthermore, SIRT3 expression in markedly elevated in melanoma compared with melanocytic nevi tissues; the same is true in cell lines derived from these tissues. Knockdown of SIRT3 reduces proliferation, colony formation, and cellular migration, and induces senescence 
and G1-phase arrest in human melanoma cells, with a corresponding increase in p16 and p21 expression and a decrease in expression of cyclin D1 and E1 and cyclin-dependent kinases (CDKs) 2, 4, and 6. SIRT3 knockdown significantly inhibits tumorigenesis in a xenograft model of melanoma. Conversely, forced overexpression of SIRT3 increases the proliferative potential of melanoma cell lines (57). SIRT3 is also overexpressed in renal cancer, in which SIRT3 depletion or an inactive mutant inhibits cell proliferation and xenograft tumor growth (58). Immunohistochemical analysis of thyroid cancer demonstrated that SIRT3 expression is associated with tumors showing low SIRT3 levels in benign thyroid tissues, moderately increased levels in follicular carcinomas, and high levels in papillary thyroid carcinomas and welldifferentiated thyroid carcinomas. Such a distribution is in accordance with the expression pattern of nicotinamide phosphoribosyltransferase (NAMPT), a rate-limiting enzyme in $\mathrm{NAD}^{+}$biosynthesis, supporting the critical involvement of the $\mathrm{NAD}^{+}$-dependent deacetylase, SIRT3, in thyroid gland (59).

SIRT3 as a tumor suppressor. SIRT3 inhibits proliferation, metabolic reprogramming, and other malignant phenotypes of cells, and correlates with a good outcome for many types of cancer. For instance, SIRT3 expression is markedly lower in breast cancer cells than in paired normal breast epithelium, and lower SIRT3 expression is associated with shorter locoregional relapse-free survival (60). SIRT3 expression also correlates with clinical characteristics such as the distribution of estrogen receptors and levels of oxidative stress (61). SIRT3 induces inhibition of glycolysis and reverses metabolic reprogramming in breast cell lines (24). The tumor-suppressive role of SIRT3 is also manifest in hepatocellular cancer (HCC), in which SIRT3 expression in cancerous tissues is much lower than that in adjacent non-cancerous tissue. Survival analyses indicate that high SIRT3 expression correlates with increased overall survival and recurrence-free survival. In addition, lower SIRT3 expression is associated with unfavorable clinicopathological parameters such as differentiation, clinical stage, and tumor multiplicity (62-64). Overexpression of SIRT3 inhibits growth and induces apoptosis in HCC cells and increases their sensitivity to chemotherapeutic agents (65-67). Furthermore, lower SIRT3 expression correlates with increased aggressiveness of pancreatic cancer and a shorter time to relapse and patient survival in the absence of chemotherapeutic intervention, suggesting that SIRT3 is tumor-suppressive in the context of pancreatic cancer and may also represent a novel predictive biomarker for chemotherapeutic response (68). Mechanistically, SIRT3 inhibits pancreatic cell metabolism and growth by impeding malate-aspartate shuttle activity (25) or preventing iron metabolism (69). SIRT3 expression in samples from patients with B cell malignancies is lower than that in primary B cells from healthy donors, and low SIRT3 expression predicts worse overall survival. Overexpression of SIRT3 decreases the proliferation and diminishes the Warburg-like phenotype of SIRT3-deficient B cell lymphoma cells (70). As a potential prognostic biomarker for basal cell cancer, SIRT3 expression is lower in cancerous tissues than in normal tissues (71). In addition, SIRT3 expression is significantly downregulated in metastatic ovarian cancer tissues and in highly metastatic ovarian cancer cell lines. Knockdown of
SIRT3 increases ovarian cancer cell migration and invasion in vitro and liver metastasis in vivo by downregulating Twist, thereby suppressing epithelial-to-mesenchymal transition (EMT) (72).

\section{Mechanisms underlying SIRT3-mediated regulation of cancer}

The precise mechanism by which SIRT3 regulates the pathogenesis of cancer remains a matter for debate. Nevertheless, depletion of ROS, modulation of metabolism, and regulation of proliferative or apoptotic pathways provide a biochemical rationale for its regulatory role in cancer (Fig. 4).

Depletion of ROS. ROS either expedite or inhibit malignancy. These controversial findings are attributed to the diverse roles played by ROS during cancer development (73). ROS can damage macromolecules like DNA by oxidizing specific intracellular chemical moieties, leading to genetic mutations and the activation of biochemical pathways that stimulate cell proliferation and neoplastic transformation (74). As shown above, SIRT3 reduces ROS levels by activating the antioxidant defense system. Loss of SIRT3 triggers oxidative damage, activates ROS-mediated signaling, and causes carcinogenesis in various cell types (14). For example, mouse embryonic fibroblasts (MEFs) lacking SIRT3 exhibit increased ROS levels and high genomic instability, and can be transformed by ectopic expression of a single oncogene. By contrast, wild-type MEFs require both Myc and Ras to acquire a malignant phenotype (75). SIRT3 knockdown facilitates tumorigenesis in xenograft models, which is inhibited by treatment with the antioxidant, $\mathrm{N}$-acetyl cysteine, whereas overexpression of SIRT3 decreases tumorigenesis in xenografts (76). Loss of SIRT3 causes MnSOD2 acetylation and elevates ROS levels, resulting in endocrine therapy resistance in human luminal $\mathrm{B}$ breast cancer (77). SIRT3 deficiency promotes the growth of B cell lymphoma via a ROS-dependent mechanism. Consistent with this, low SIRT3 levels in clinical B lymphomas correlated with hyperacetylation and reduced activities of the mitochondrial proteins IDH2 and MnSOD2 and with high levels of ROS (70). Therefore, SIRT3 functions as a tumor suppressor by scavenging ROS.

Consistent with the role of ROS in tumor formation and progression, removal of ROS may also underlie the oncogenic role of SIRT3. For example, overexpression of SIRT3 in gastric cancer promotes cell proliferation and glycolysis, which is ascribed to the ability of SIRT3 to maintain the low levels of intracellular ROS (46). Interestingly, SIRT3 activates hypoxia-induced mitophagy in human glioma cells by increasing the interaction between VDAC1 and Parkin, while inhibiting SIRT3-mediated mitophagy further decreases the mitochondrial membrane potential, and increases the accumulation of ROS, which in turn triggers degradation of the antiapoptotic proteins Mcl-1 and survivin via the proteasomal pathway; this suggests that SIRT3 may maintain ROS at levels that contribute to the malignant phenotype of cancer cells (78).

Modulation of metabolism reprogramming. Cancer cells prefer to utilize glycolysis for energy production, even in the presence of sufficient oxygen: this phenomenon is 
called 'aerobic glycolysis' or 'the Warburg effect' (79). The resulting metabolic reprogramming, in addition to enabling rapid energy supply, provides enough glycolytic intermediates for biosynthetic pathways and facilitates the synthesis of the macromolecules and organelles needed for generating new cancer cells (80-82). Accumulating studies support a suppressive role for SIRT3 in cancer-related metabolic reprogramming via two different pathways (13). First, SIRT3-mediated reductions in ROS levels induce activation of oxygen-dependent prolyl hydroxylases (PHD), followed by degradation of hypoxia-inducible factor 1- $\alpha$ (HIF-1 $\alpha$ ), a key mediator of metabolic reprogramming. Consistent with this, loss of SIRT3 from breast cancer cells provides an impetus for metabolic reprogramming via the ROS/HIF-1 $\alpha$ pathway (83). In this regard, the anticancer role of profilin1 (Pfn1) in pancreatic cancer was ascribed to its capacity to upregulate SIRT3, resulting in destabilization of HIF-1 $\alpha$ and suppressed expression of glycolytic genes (84). Second, SIRT3 stimulates the TCA or inhibits glycolysis by directly targeting metabolic enzymes. As described above, SIRT3 blocks a critical step of glycolysis in both breast cancer and gastric cancer by inhibiting HK2 $(24,45)$. Another key enzyme modified by SIRT3 is PDC, which links the glycolysis metabolic pathway to the TCA by converting pyruvate into acetyl-CoA. The activity of PDC is either inhibited by pyruvate dehydrogenase kinase or restored by pyruvate dehydrogenase phosphatase. In cancer cells, mitochondrial ACAT1 acetylates and inhibits PDC by recruiting PDK1, while PDC is deacetylated by SIRT3. ACAT1 and SIRT3 also acetylate and deacetylate PDP1, resulting in the dissociation from, or association with, PDC, respectively $(28,85)$. In pancreatic cells, SIRT3 deacetylates and inhibits the activity of GOT2, the limiting enzyme in the malate-aspartate shuttle, thereby impairing the transport of cytosolic NADH into the mitochondria, a process required for glycolysis (25).

SIRT3 also shows a dark side when regulating metabolic reprogramming. For example, in gastric cancer cells, SIRT3 can deacetylate and activate lactate dehydrogenase A, thereby promoting anaerobic glycolysis and carcinogenesis (46). In addition, a cluster of glycolysis-associated proteins (HK2 and glucose transporters) is upregulated in SIRT3-expressing gastric carcinoma cells (46). These findings suggest that SIRT3 may expedite malignant transformation by promoting metabolic reprogramming.

Control of proliferative signaling. Cell division is driven by intracellular signals normally initiated by growth factor engagement of cognate receptors, culminating in activation of transcriptional factors and expression of proteins required for cell cycle progression. The canonical signaling pathways that contribute to cell proliferation are tailored and orchestrated by numerous regulators. To this end, SIRT3 deacetylates and modifies the activity of components or master regulators of proliferative signaling and is, therefore, essential for tumorigenesis.

First, SIRT3 plays a regulatory role in cellular levels of P53, the well-characterized tumor suppressor. SIRT3 attenuates p53 degradation by downregulating the mouse double minute 2 homolog (MDM2) in HCC cells (65). SIRT3-mediated deacetylation also upregulates the activity of phosphatase and tension homolog, leading to a reduction in MDM2 transcription and p53 degradation in various cancer cells $(48,86)$.

Second, SIRT3 dictates cell proliferation by modulating the classical mitogen-activated protein kinases/extracellular signal-regulated kinases (MAPK/ERK) and protein kinase B (PKB/Akt) signaling pathways. In HCC and prostate cancer cells, ectopic expression of SIRT3 reduces phosphorylation of ERK1/2 and Akt, thereby suppressing cell survival and proliferation (65). While reduced mitochondrial ROS production may underlie SIRT3 suppression of the PI3K/Akt pathway, the ubiquitination and degradation of the oncoprotein c-MYC is responsible for inhibiting cell proliferation downstream of SIRT3/Akt signaling $(87,88)$.

Third, SIRT3 can suppress mitosis by directly targeting cell cycle proteins. S-phase kinase-associated protein 2 (Skp2) enables cell cycle progression through the G1/S checkpoint by promoting destruction of p27 (89). Acetylation of Skp2 by p300 increases its stability and cytoplasmic translocation, whereas SIRT3 deacetylates Skp2 and antagonizes p300-mediated Skp2 activation (90).

Finally, SIRT3 suppresses the growth of pancreatic cancer by modulating cellular iron metabolism. Overexpression of SIRT3 impairs enrichment of iron regulatory protein 1 (IRP1) on the iron-responsive elements, thereby downregulating the transferrin receptor 1 (TfR1) and suppressing TfR1-mediated iron uptake and cell growth (69).

It is noteworthy that SIRT3 also facilitates carcinogenesis by reinforcing proliferative signal pathways. In bladder tumorderived EJ-P53 cells, SIRT3 appears to rescue p53-induced growth arrest by abrogating p53 activity (91). In non-small cell lung cancer cells, SIRT3 promotes growth and proliferation by interacting with nicotinamide mononucleotide adenylyltransferase 2 (NMNAT2), which catalyzes an essential step in NAD (and NADP) biosynthesis (47).

Regulation of apoptotic pathways. Programmed cell death or apoptosis plays an important role in ontogenesis and the development of various disorders. Resistance to apoptosis is a hallmark of cancer cells (2). Recent studies show that SIRT3 extensively participates in the regulation of the apoptotic machinery.

The B cell lymphoma 2 (Bcl-2) family proteins are key regulators of the apoptotic pathway. Its proapoptotic members, such as Bcl-2-associated X protein (Bax), damage the integrity of the outer mitochondrial membrane, leading to cytochrome $c$ release into the cytosol and a subsequent cascade of caspase activation that triggers apoptosis. The mitochondrial poreforming activity of these proapoptotic proteins is inhibited by their association with antiapoptotic members. SIRT3 regulates both proapoptotic and antiapoptotic members of the Bcl-2 family. For example, SIRT3 mediates Bcl-2- and JNK2-regulated apoptosis in colorectal carcinoma cells under basal conditions (92). Moreover, SIRT3 overexpression in HCC cells promotes chemotherapeutic agent- and sorafenibinduced apoptosis, while SIRT3 silencing confers resistance to chemotherapy. Mechanistically, SIRT3 decreases the amount of glutathione S-transferase P1 (GSTP1), causing sequential activation of JNK and c-Jun and upregulating the c-Jun transcriptional target, Bim (67). The proapoptotic role of SIRT3 was also verified in lung adenocarcinoma cells, in which SIRT3 
overexpression induces apoptosis by increasing the Bax/ Bcl-2 ratio (48). Kaempferol, a flavonoid compound, induces apoptosis by upregulating Bax and SIRT3 and downregulating $\mathrm{Bcl}-2$ (93). Interestingly, the $\mathrm{BH} 3$-only protein mimetic $\mathrm{S} 1$, a novel pan Bcl-2 inhibitor, simultaneously interrupts glucose metabolism and induces apoptosis of ovarian cancer cells by upregulating and inducing the mitochondrial translocation of nuclear SIRT3, suggesting reciprocal regulation between SIRT3 and Bcl-2 family proteins in cancer cells (94). Finally, given the regulatory role of SIRT3 on p53, SIRT3 may also induce apoptosis through transcriptional activation of Bax and downregulation of Bcl-2.

Conversely, SIRT3 could also function as an antiapoptotic protein in various types of cell. SIRT3 blocks apoptosis in cervical carcinoma HeLa cells by deacetylating $\mathrm{Ku} 70$, thereby facilitating its association with Bax and preventing Bax translocation to the mitochondrion (11). Cancer cells have evolved the ability to escape anoikis, a specific form of apoptosis triggered by loss of contact with the extracellular matrix $(95,96)$. In oral squamous cell carcinoma, dissociation of focal adhesion kinase from receptor interacting protein (RIP) promotes RIP binding to Fas and the formation of the death-inducing signaling complex that triggers anoikis; however, SIRT3 functions as a negative regulator of RIP downstream signaling, thereby rendering neoplastic cells resistant to anoikis $(97,98)$.

\section{Conclusion}

As a crucial mitochondrial deacetylase, SIRT3 modulates the activity of an increasing list of substrate proteins. Mounting evidence supports causal involvement of SIRT3 in various disorders, including carcinogenesis. However, studies using different carcinoma models have reached diverse conclusions concerning the precise role of SIRT3 in tumorigenesis. These studies may have reached different conclusions because SIRT3 acts on a wide array of substrates in different cellular contexts. Also, SIRT3 catalyzes deacetylation of both histone and nonhistone proteins in the mitochondrion and nucleus, which has different effects on individual proteins. These are further complicated by the non-physiological manipulation of genes in vitro. Therefore, further studies are required to discriminate SIRT3-regulated key events that drive carcinogenesis from those that do not (or even suppress it). In this respect, while utilization of SIRT3-deficient cells or animal models allows systemic study of its global contributions to intracellular signaling or metabolic pattern shifts, studies based on clinical cancers will help define a bona fide correlation between SIRT3 and the pathogenesis of different malignancies. Nonetheless, a clearer understanding of the role of SIRT3 in carcinogenesis will eventually open new avenues for cancer treatment by targeting this mitochondrial deacetylase.

\section{References}

1. Jemal A, Bray F, Center MM, Ferlay J, Ward E and Forman D: Global cancer statistics. CA Cancer J Clin 61: 69-90, 2011.

2. Hanahan D and Weinberg RA: Hallmarks of cancer: The next generation. Cell 144: 646-674, 2011

3. Choudhary C, Kumar C, Gnad F, Nielsen ML, Rehman M, Walther TC, Olsen JV and Mann M: Lysine acetylation targets protein complexes and co-regulates major cellular functions. Science 325: 834-840, 2009.
4. Kim SC, Sprung R, Chen Y, Xu Y, Ball H, Pei J, Cheng T, Kho Y, Xiao H, Xiao L, et al: Substrate and functional diversity of lysine acetylation revealed by a proteomics survey. Mol Cell 23: 607-618, 2006

5. Guan KL and Xiong Y: Regulation of intermediary metabolism by protein acetylation. Trends Biochem Sci 36: 108-116, 2011.

6. Frye RA: Phylogenetic classification of prokaryotic and eukaryotic Sir2-like proteins. Biochem Biophys Res Commun 273: 793-798, 2000

7. Haigis MC and Guarente LP: Mammalian sirtuins - emerging roles in physiology, aging, and calorie restriction. Genes Dev 20: 2913-2921, 2006

8. Weir HJ, Lane JD and Balthasar N: SIRT3: A central regulator of mitochondrial adaptation in health and disease. Genes Cancer 4: 118-124, 2013.

9. Hallows WC, Albaugh BN and Denu JM: Where in the cell is SIRT3? - functional localization of an $\mathrm{NAD}^{+}$-dependent protein deacetylase. Biochem J 411: e11-e13, 2008.

10. Jing E, Emanuelli B, Hirschey MD, Boucher J, Lee KY, Lombard D, Verdin EM and Kahn CR: Sirtuin-3 (Sirt3) regulates skeletal muscle metabolism and insulin signaling via altered mitochondrial oxidation and reactive oxygen species production. Proc Natl Acad Sci USA 108: 14608-14613, 2011.

11. Sundaresan NR, Samant SA, Pillai VB, Rajamohan SB and Gupta MP: SIRT3 is a stress-responsive deacetylase in cardiomyocytes that protects cells from stress-mediated cell death by deacetylation of Ku70. Mol Cell Biol 28: 6384-6401, 2008.

12. Kumar S and Lombard DB: Mitochondrial sirtuins and their relationships with metabolic disease and cancer. Antioxid Redox Signal 22: 1060-1077, 2015.

13. Haigis MC, Deng C-X, Finley LWS, Kim H-S and Gius D: SIRT3 is a mitochondrial tumor suppressor: A scientific tale that connects aberrant cellular ROS, the Warburg effect, and carcinogenesis. Cancer Res 72: 2468-2472, 2012.

14. Finley LWS and Haigis MC: Metabolic regulation by SIRT3: Implications for tumorigenesis. Trends Mol Med 18: 516-523, 2012.

15. Alhazzazi TY, Kamarajan P, Verdin E and Kapila YL: Sirtuin-3 (SIRT3) and the hallmarks of cancer. Genes Cancer 4: 164-171, 2013.

16. Chen Y, Fu LL, Wen X, Wang XY, Liu J, Cheng Y and Huang J: Sirtuin-3 (SIRT3), a therapeutic target with oncogenic and tumorsuppressive function in cancer. Cell Death Dis 5: e1047, 2014.

17. Nguyen GT, Schaefer S, Gertz M, Weyand M and Steegborn C: Structures of human sirtuin 3 complexes with ADP-ribose and with carba-NAD ${ }^{+}$and SRT1720: Binding details and inhibition mechanism. Acta Crystallogr D Biol Crystallogr 69: 1423-1432, 2013.

18. Jin L, Wei W, Jiang Y, Peng H, Cai J, Mao C, Dai H, Choy W, Bemis JE, Jirousek MR, et al: Crystal structures of human SIRT3 displaying substrate-induced conformational changes. J Biol Chem 284: 24394-24405, 2009.

19. Schwer B, North BJ, Frye RA, Ott M and Verdin E: The human silent information regulator (Sir)2 homologue hSIRT3 is a mitochondrial nicotinamide adenine dinucleotide-dependent deacetylase. J Cell Biol 158: 647-657, 2002.

20. Cooper HM, Huang JY, Verdin E and Spelbrink JN: A new splice variant of the mouse SIRT3 gene encodes the mitochondrial precursor protein. PLoS One 4: e4986, 2009.

21. Onyango P, Celic I, McCaffery JM, Boeke JD and Feinberg AP: SIRT3, a human SIR2 homologue, is an NAD-dependent deacetylase localized to mitochondria. Proc Natl Acad Sci USA 99: 13653-13658, 2002.

22. Scher MB, Vaquero A and Reinberg D: SirT3 is a nuclear $\mathrm{NAD}^{+}$-dependent histone deacetylase that translocates to the mitochondria upon cellular stress. Genes Dev 21: 920-928, 2007.

23. Ahn B-H, Kim H-S, Song S, Lee IH, Liu J, Vassilopoulos A, Deng CX and Finkel T: A role for the mitochondrial deacetylase Sirt3 in regulating energy homeostasis. Proc Natl Acad Sci USA 105: 14447-14452, 2008

24. Wei L, Zhou Y, Dai Q, Qiao C, Zhao L, Hui H, Lu N and Guo QL: Oroxylin A induces dissociation of hexokinase II from the mitochondria and inhibits glycolysis by SIRT3-mediated deacetylation of cyclophilin D in breast carcinoma. Cell Death Dis 4: e601, 2013.

25. Yang H, Zhou L, Shi Q, Zhao Y, Lin H, Zhang M, Zhao S, Yang Y, Ling ZQ, Guan KL, et al: SIRT3-dependent GOT2 acetylation status affects the malate-aspartate NADH shuttle activity and pancreatic tumor growth. EMBO J 34: 1110-1125, 2015. 
26. Hallows WC, Lee S and Denu JM: Sirtuins deacetylate and activate mammalian acetyl-CoA synthetases. Proc Natl Acad Sci USA 103: 10230-10235, 2006.

27. Schwer B, Bunkenborg J, Verdin RO, Andersen JS and Verdin E: Reversible lysine acetylation controls the activity of the mitochondrial enzyme acetyl-CoA synthetase 2. Proc Natl Acad Sci USA 103: 10224-10229, 2006.

28. Shan C, Kang H-B, Elf S, Xie J, Gu TL, Aguiar M, Lonning S, Hitosugi T, Chung TW, Arellano M, et al: Tyr-94 phosphorylation inhibits pyruvate dehydrogenase phosphatase 1 and promotes tumor growth. J Biol Chem 289: 21413-21422, 2014.

29. Someya S, Yu W, Hallows WC, Xu J, Vann JM, Leeuwenburgh C, Tanokura M, Denu JM and Prolla TA: Sirt3 mediates reduction of oxidative damage and prevention of age-related hearing loss under caloric restriction. Cell 143: 802-812, 2010.

30. Cimen H, Han MJ, Yang Y, Tong Q, Koc H and Koc EC: Regulation of succinate dehydrogenase activity by SIRT3 in mammalian mitochondria. Biochemistry 49: 304-311, 2010.

31. Finley LW, Haas W, Desquiret-Dumas V, Wallace DC, Procaccio V, Gygi SP and Haigis MC: Succinate dehydrogenase is a direct target of sirtuin 3 deacetylase activity. PLoS One 6 : e23295, 2011.

32. Schlicker C, Gertz M, Papatheodorou P, Kachholz B, Becker CF and Steegborn C: Substrates and regulation mechanisms for the human mitochondrial sirtuins Sirt3 and Sirt5. J Mol Biol 382: 790-801, 2008

33. Hallows WC, Yu W, Smith BC, Devries MK, Ellinger JJ, Someya S, Shortreed MR, Prolla T, Markley JL, Smith LM, et al: Sirt3 promotes the urea cycle and fatty acid oxidation during dietary restriction. Mol Cell 41: 139-149, 2011.

34. Hirschey MD, Shimazu T, Goetzman E, Jing E, Schwer B, Lombard DB, Grueter CA, Harris C, Biddinger S, Ilkayeva OR, et al: SIRT3 regulates mitochondrial fatty-acid oxidation by reversible enzyme deacetylation. Nature 464: 121-125, 2010.

35. Shimazu T, Hirschey MD, Hua L, Dittenhafer-Reed KE, Schwer B, Lombard DB, Li Y, Bunkenborg J, Alt FW, Denu JM, et al: SIRT3 deacetylates mitochondrial 3-hydroxy-3-methylglutaryl CoA synthase 2 and regulates ketone body production. Cell Metab 12: 654-661, 2010.

36. Sundaresan NR, Gupta M, Kim G, Rajamohan SB, Isbatan A and Gupta MP: Sirt3 blocks the cardiac hypertrophic response by augmenting Foxo3a-dependent antioxidant defense mechanisms in mice. J Clin Invest 119: 2758-2771, 2009.

37. Pillai VB, Sundaresan NR, Kim G, Gupta M, Rajamohan SB, Pillai JB, Samant S, Ravindra PV, Isbatan A and Gupta MP. Exogenous NAD blocks cardiac hypertrophic response via activation of the SIRT3-LKB1-AMP-activated kinase pathway. J Biol Chem 285: 3133-3144, 2010.

38. Cantó C, Gerhart-Hines Z, Feige JN, Lagouge M, Noriega L, Milne JC, Elliott PJ, Puigserver P and Auwerx J: AMPK regulates energy expenditure by modulating NAD ${ }^{+}$metabolism and SIRT 1 activity. Nature 458: 1056-1060, 2009.

39. Yang H, Yang T, Baur JA, Perez E, Matsui T, Carmona JJ, Lamming DW, Souza-Pinto NC, Bohr VA, Rosenzweig A et al: Nutrient-sensitive mitochondrial $\mathrm{NAD}^{+}$levels dictate cell survival. Cell 130: 1095-1107, 2007.

40. Cheng Y, Ren X, Gowda ASP, Shan Y, Zhang L, Yuan YS Patel R, Wu H, Huber-Keener K, Yang JW, et al: Interaction of Sirt3 with OGG1 contributes to repair of mitochondrial DNA and protects from apoptotic cell death under oxidative stress. Cell Death Dis 4: e731, 2013.

41. Yang Y, Cimen H, Han MJ, Shi T, Deng JH, Koc H, Palacios OM, Montier L, Bai Y, Tong Q, et al: NAD ${ }^{+}$-dependent deacetylase SIRT3 regulates mitochondrial protein synthesis by deacetylation of the ribosomal protein MRPL10. J Biol Chem 285: 7417-7429, 2010.

42. Samant SA, Zhang HJ, Hong Z, Pillai VB, Sundaresan NR, Wolfgeher D, Archer SL, Chan DC and Gupta MP: SIRT3 deacetylates and activates OPA1 to regulate mitochondrial dynamics during stress. Mol Cell Biol 34: 807-819, 2014.

43. Shulga $\mathrm{N}$ and Pastorino JG: Ethanol sensitizes mitochondria to the permeability transition by inhibiting deacetylation of cyclophilin-D mediated by sirtuin-3. J Cell Sci 123: 4117-4127, 2010.

44. Papa L and Germain D: SirT3 regulates the mitochondrial unfolded protein response. Mol Cell Biol 34: 699-710, 2014.

45. Yang B, Fu X, Shao L, Ding Y and Zeng D: Aberrant expression of SIRT3 is conversely correlated with the progression and prognosis of human gastric cancer. Biochem Biophys Res Commun 443: 156-160, 2014
46. Cui Y, Qin L, Wu J, Qu X, Hou C, Sun W, Li S, Vaughan AT, Li JJ and Liu J: SIRT3 enhances glycolysis and proliferation in SIRT3-expressing gastric cancer cells. PLoS One 10: e0129834, 2015.

47. Li H, Feng Z, Wu W, Li J, Zhang J and Xia T: SIRT3 regulates cell proliferation and apoptosis related to energy metabolism in non-small cell lung cancer cells through deacetylation of NMNAT2. Int J Oncol 43: 1420-1430, 2013.

48. Xiao K, Jiang J, Wang W, Cao S, Zhu L, Zeng H, Ouyang R, Zhou R and Chen P: Sirt3 is a tumor suppressor in lung adenocarcinoma cells. Oncol Rep 30: 1323-1328, 2013.

49. Liang L, Li Q, Huang L, Li D and Li X: Sirt3 binds to and deacetylates mitochondrial pyruvate carrier 1 to enhance its activity. Biochem Biophys Res Commun 468: 807-812, 2015.

50. Liu C, Huang Z, Jiang $\mathrm{H}$ and Shi F: The sirtuin 3 expression profile is associated with pathological and clinical outcomes in colon cancer patients. Biomed Res Int 2014: 871263-871263, 2014.

51. Lai C-C, Lin P-M, Lin S-F, Hsu CH, Lin HC, Hu ML, Hsu CM and Yang MY: Altered expression of SIRT gene family in head and neck squamous cell carcinoma. Tumour Biol 34: 1847-1854, 2013.

52. Mahjabeen I and Kayani MA: Loss of mitochondrial tumor suppressor genes expression is associated with unfavorable clinical outcome in head and neck squamous cell carcinoma: Data from retrospective study. PLoS One 11: e0146948, 2016.

53. Alhazzazi TY, Kamarajan P, Xu Y, Ai T, Chen L, Verdin E and Kapila YL: A novel sirtuin-3 inhibitor, LC-0296, inhibits cell survival and proliferation, and promotes apoptosis of head and neck cancer cells. Anticancer Res 36: 49-60, 2016.

54. Zhao Y, Yang H, Wang X, Zhang R, Wang C and Guo Z: Sirtuin-3 (SIRT3) expression is associated with overall survival in esophageal cancer. Ann Diagn Pathol 17: 483-485, 2013.

55. Torrens-Mas M, Pons DG, Sastre-Serra J, Oliver J and Roca P: SIRT3 silencing sensitizes breast cancer cells to cytotoxic treatments through an increment in ROS production. J Cell Biochem: Jul 15, 2016 (Epub ahead of print). doi: 10.1002/jcb.25653.

56. Alhazzazi TY, Kamarajan P, Joo N, Huang JY, Verdin E, D'Silva NJ and Kapila YL: Sirtuin-3 (SIRT3), a novel potential therapeutic target for oral cancer. Cancer 117: 1670-1678, 2011.

57. George J, Nihal M, Singh CK, Zhong W, Liu X and Ahmad N: Pro-proliferative function of mitochondrial sirtuin deacetylase SIRT3 in human melanoma. J Invest Dermatol 136: 809-818, 2016.

58. Choi J, Koh E, Lee YS, Lee HW, Kang HG, Yoon YE, Han WK, Choi KH and Kim KS: Mitochondrial Sirt3 supports cell proliferation by regulating glutamine-dependent oxidation in renal cell carcinoma. Biochem Biophys Res Commun 474: 547-553, 2016.

59. Shackelford R, Hirsh S, Henry K, Abdel-Mageed A, Kandil E and Coppola D: Nicotinamide phosphoribosyltransferase and SIRT3 expression are increased in well-differentiated thyroid carcinomas. Anticancer Res 33: 3047-3052, 2013.

60. Desouki MM, Doubinskaia I, Gius D and Abdulkadir SA: Decreased mitochondrial SIRT3 expression is a potential molecular biomarker associated with poor outcome in breast cancer. Hum Pathol 45: 1071-1077, 2014.

61. Sastre-Serra J, Nadal-Serrano M, Pons DG, Valle A, Garau I, García-Bonafé M, Oliver J and Roca P: The oxidative stress in breast tumors of postmenopausal women is $E R \alpha / E R \beta$ ratio dependent. Free Radic Biol Med 61: 11-17, 2013.

62. Zhang CZ, Liu L, Cai M, Pan Y, Fu J, Cao Y and Yun J: Low SIRT3 expression correlates with poor differentiation and unfavorable prognosis in primary hepatocellular carcinoma. PLoS One 7: e51703, 2012.

63. Zhang B, Qin L, Zhou C-J, Liu Y-L, Qian H-X and He S-B SIRT3 expression in hepatocellular carcinoma and its impact on proliferation and invasion of hepatoma cells. Asian Pac J Trop Med 6: 649-652, 2013.

64. Wang J-X, Yi Y, Li Y-W, Cai XY, He HW, Ni XC, Zhou J, Cheng YF, Jin JJ, Fan J, et al: Down-regulation of sirtuin 3 is associated with poor prognosis in hepatocellular carcinoma after resection. BMC Cancer 14: 297, 2014.

65. Zhang Y-Y and Zhou L-M: Sirt3 inhibits hepatocellular carcinoma cell growth through reducing Mdm2-mediated p53 degradation. Biochem Biophys Res Commun 423: 26-31, 2012.

66. Li Y, Wang W, Xu X, Sun S and Qu XJ: \{2-[1- (3-methoxycarbonylmethyl-1H-indol-2-yl)-1-methyl-ethyl]-1H-indol-3-yl $\}$-acetic acid methyl ester (MIAM) inhibited human hepatocellular carcinoma growth through upregulation of Sirtuin-3 (SIRT3). Biomed Pharmacother 69: 125-132, 2015. 
67. Tao NN, Zhou HZ, Tang H, Cai XF, Zhang WL, Ren JH, Zhou L, Chen X, Chen K, Li WY, et al: Sirtuin 3 enhanced drug sensitivity of human hepatoma cells through glutathione S-transferase pi 1/JNK signaling pathway. Oncotarget 7: 50117-50130, 2016.

68. McGlynn LM, McCluney S, Jamieson NB, Thomson J, MacDonald AI, Oien K, Dickson EJ, Carter CR, McKay CJ and Shiels PG: SIRT3 \& SIRT7: Potential novel biomarkers for determining outcome in pancreatic cancer patients. PLoS One 10: e0131344, 2015

69. Jeong SM, Lee J, Finley LWS, Schmidt PJ, Fleming MD and Haigis MC: SIRT3 regulates cellular iron metabolism and cancer growth by repressing iron regulatory protein 1 . Oncogene 34 2115-2124, 2015

70. Yu W, Denu RA, Krautkramer KA, Grindle KM, Yang DT, Asimakopoulos F, Hematti P and Denu JM: Loss of SIRT3 provides growth advantage for B cell malignancies. J Biol Chem 291: 3268-3279, 2016.

71. Temel M, Koc MN, Ulutas S and Gogebakan B: The expression levels of the sirtuins in patients with BCC. Tumour Biol 37: 6429-6435, 2016.

72. Dong XC, Jing LM, Wang WX and Gao YX: Down-regulation of SIRT3 promotes ovarian carcinoma metastasis. Biochem Biophys Res Commun 475: 245-250, 2016.

73. Chandel NS and Tuveson DA: The promise and perils of antioxidants for cancer patients. N Engl J Med 371: 177-178, 2014

74. Finkel T: Signal transduction by reactive oxygen species. J Cell Biol 194: 7-15, 2011

75. Kim H-S, Patel K, Muldoon-Jacobs K, Bisht KS, Aykin-Burns N, Pennington JD, van der Meer R, Nguyen P, Savage J, Owens KM, et al: SIRT3 is a mitochondria-localized tumor suppressor required for maintenance of mitochondrial integrity and metabolism during stress. Cancer Cell 17: 41-52, 2010.

76. Bell EL, Emerling BM, Ricoult SJH and Guarente L: SirT3 suppresses hypoxia inducible factor $1 \alpha$ and tumor growth by inhibiting mitochondrial ROS production. Oncogene 30 : 2986-2996, 2011.

77. Zou X, Santa-Maria CA, O'Brien J, Gius D and Zhu Y: Manganese superoxide dismutase acetylation and dysregulation, due to loss of SIRT3 activity, promote a luminal B-like breast carcinogenicpermissive phenotype. Antioxid Redox Signal 25: 326-336, 2016.

78. Qiao A, Wang K, Yuan Y, Guan Y, Ren X, Li L, Chen X, Li F, Chen AF, Zhou J, et al: Sirt3-mediated mitophagy protects tumor cells against apoptosis under hypoxia. Oncotarget: May 30, 2016 (Epub ahead of print). doi: 10.18632/oncotarget.9717.

79. Warburg O: On the origin of cancer cells. Science 123: 309-314, 1956.

80. Vander Heiden MG, Cantley LC and Thompson CB: Understanding the Warburg effect: The metabolic requirements of cell proliferation. Science 324: 1029-1033, 2009.

81. DeBerardinis RJ, Lum JJ, Hatzivassiliou G and Thompson CB The biology of cancer: Metabolic reprogramming fuels cell growth and proliferation. Cell Metab 7: 11-20, 2008

82. Christofk HR, Vander Heiden MG, Harris MH, Ramanathan A, Gerszten RE, Wei R, Fleming MD, Schreiber SL and Cantley LC: The M2 splice isoform of pyruvate kinase is important for cancer metabolism and tumour growth. Nature 452: 230-233, 2008.

83. Wei L, Zhou Y, Qiao C, Ni T, Li Z, You Q, Guo Q and Lu N: Oroxylin A inhibits glycolysis-dependent proliferation of human breast cancer via promoting SIRT3-mediated SOD2 transcription and HIF1 $\alpha$ destabilization. Cell Death Dis 6: e1714, 2015.
84. Yao W, Ji S, Qin Y, Yang J, Xu J, Zhang B, Xu W, Liu J, Shi S, Liu L, et al: Profilin-1 suppresses tumorigenicity in pancreatic cancer through regulation of the SIRT3-HIF1 $\alpha$ axis. Mol Cancer 13: 187, 2014.

85. Fan J, Shan C, Kang HB, Elf S, Xie J, Tucker M, Gu TL, Aguiar M, Lonning S, Chen $\mathrm{H}$, et al: Tyr phosphorylation of PDP1 toggles recruitment between ACAT1 and SIRT3 to regulate the pyruvate dehydrogenase complex. Mol Cell 53: 534-548, 2014.

86. Zhao K, Zhou Y, Qiao C, Ni T, Li Z, Wang X, Guo Q, Lu N and Wei L: Oroxylin A promotes PTEN-mediated negative regulation of MDM2 transcription via SIRT3-mediated deacetylation to stabilize p53 and inhibit glycolysis in wt-p53 cancer cells. J Hematol Oncol 8: 41, 2015.

87. Quan Y, Wang N, Chen Q, Xu J, Cheng W, Di M, Xia W and Gao WQ: SIRT3 inhibits prostate cancer by destabilizing oncoprotein c-MYC through regulation of the PI3K/Akt pathway. Oncotarget 6: 26494-26507, 2015.

88. Pillai VB, Sundaresan NR and Gupta MP: Regulation of Akt signaling by sirtuins: Its implication in cardiac hypertrophy and aging. Circ Res 114: 368-378, 2014.

89. Carrano AC, Eytan E, Hershko A and Pagano M: SKP2 is required for ubiquitin-mediated degradation of the $\mathrm{CDK}$ inhibitor p27. Nat Cell Biol 1: 193-199, 1999.

90. Wang Z, Inuzuka H, Zhong J, Liu P, Sarkar FH, Sun Y and Wei W: Identification of acetylation-dependent regulatory mechanisms that govern the oncogenic functions of Skp2. Oncotarget 3: 1294-1300, 2012.

91. Li S, Banck M, Mujtaba S, Zhou M-M, Sugrue MM and Walsh MJ: p53-induced growth arrest is regulated by the mitochondrial SirT3 deacetylase. PLoS One 5: e10486, 2010.

92. Allison SJ and Milner J: SIRT3 is pro-apoptotic and participates in distinct basal apoptotic pathways. Cell Cycle 6: 2669-2677, 2007.

93. Marfe G, Tafani M, Indelicato M, Sinibaldi-Salimei P, Reali V, Pucci B, Fini M and Russo MA: Kaempferol induces apoptosis in two different cell lines via Akt inactivation, Bax and SIRT3 activation, and mitochondrial dysfunction. J Cell Biochem 106: 643-650, 2009.

94. Xiang XY, Kang JS, Yang XC, Su J, Wu Y, Yan XY, Xue YN, $\mathrm{Xu}$ Y, Liu YH, Yu CY, et al: SIRT3 participates in glucose metabolism interruption and apoptosis induced by $\mathrm{BH} 3$ mimetic S1 in ovarian cancer cells. Int J Oncol 49: 773-784, 2016.

95. Shin SI, Freedman VH, Risser R and Pollack R: Tumorigenicity of virus-transformed cells in nude mice is correlated specifically with anchorage independent growth in vitro. Proc Natl Acad Sci USA 72: 4435-4439, 1975.

96. Kantak SS and Kramer RH: E-cadherin regulates anchorageindependent growth and survival in oral squamous cell carcinoma cells. J Biol Chem 273: 16953-16961, 1998.

97. Kamarajan P, Bunek J, Lin Y, Nunez G and Kapila YL: Receptorinteracting protein shuttles between cell death and survival signaling pathways. Mol Biol Cell 21: 481-488, 2010.

98. Kamarajan P, Alhazzazi TY, Danciu T, D'silva NJ, Verdin E and Kapila YL: Receptor-interacting protein (RIP) and Sirtuin-3 (SIRT3) are on opposite sides of anoikis and tumorigenesis. Cancer 118: 5800-5810, 2012. 